\title{
Canadians leading anti-Ebola research
}

$\mathrm{T}$ he West African Ebola epidemic shows no sign of slowing, but this outbreak - the largest and most lethal on record - may also be the last without treatment options.

Canadian scientists and drug companies are among those closest to developing a treatment for the hemorrhagic fever. Currently, there is no vaccine or treatment for Ebola.

The virus has infected 1093 people and killed 660 in Sierra Leone, Guinea and Liberia since the outbreak began in January, according to a July 20 tally by the World Health Organization. This month, Médecins Sans Frontières emergency coordinator Anja Wolz suggested that these numbers may represent only the "tip of the iceberg."

Without a cure, medical workers focus on the quarantine and comfort of infected patients. With rehydration and intensive supportive care, some survive, but for a majority — up to $90 \%$ in some outbreaks - Ebola is fatal, usually within six to nine weeks after the onset of symptoms.

Transmission is through contact with blood or body fluid from infected patients or monkeys, made easier by one of the more gruesome symptoms of the illness - bleeding from the eyes, ears, nose, mouth and rectum. Initial symptoms include sudden onset of fever and sore throat, followed by vomiting, diarrhea and impaired kidney and liver function.

Recent strides in the development of two anti-Ebola drugs in Canada have sparked some optimism, but major hurdles remain to bring these treatments to the field.

In March, the United States Food and Drug Administration (FDA) expedited the review of TKM-Ebola, a postexposure prophylactic drug developed by Vancouver-based Tekmira Pharmaceuticals Corporation. In preclinical studies published in The Lancet May 29,2010 , two out of three rhesus monkeys given four postexposure treatments and three out of three macaques

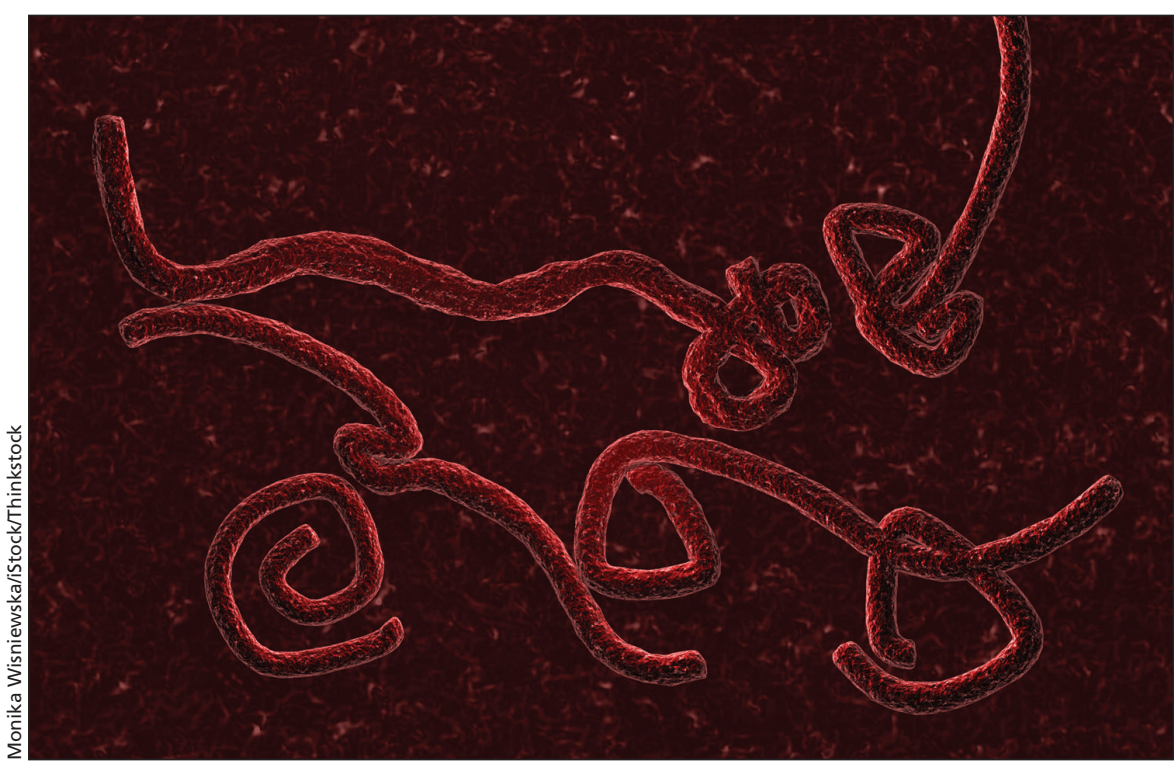

Canadian researchers are among those closest to a postexposure treatment for Ebola.

given seven postexposure treatments were protected from Ebola.

But in July, the FDA put phase 1 clinical trials of the drug on hold over concerns about the dose being given to volunteers. The delay drew criticism from global health experts, including Jeremy Farrar, director of the Wellcome Trust, an international medical research charity based in the United Kingdom. More than 6000 people have since petitioned the FDA to release the hold on TKM-Ebola trials and fast track other drug and vaccine research.

On July 21, Tekmira said it was preparing a response to the FDA's hold letter and hopes to resume trials of TKM-Ebola later this year.

Canada's National Microbiology Laboratory is developing a second potential treatment in partnership with Toronto-based biodefense company Defyrus Inc. and Mapp Biopharmaceutical Inc. in the US.

Called ZMapp, the drug is a cocktail of three antibodies that target Ebola's protective protein envelope, and has shown promising results in studies of monkeys. In preclinical trials published in Science Translational Medicine on June 13, 2012, all four macaques treated with this cocktail within 24 hours after exposure to Ebola, and two of four treated within 48 hours, survived.

To be truly useful in the field, however, postexposure treatments for Ebola need to be effective when administered several days after the initial infection, because symptoms aren't always immediately apparent.

A small study of eight monkeys that was published in Science Translational Medicine Oct. 16, 2013, tested a new formulation of ZMapp, designed to extend the treatment window. Administered three days after the monkeys were exposed to Ebola, the new cocktail saved all but one. Scientists at the National Microbiology Laboratory expect to begin human testing in the first half of 2015.

Although the drug won't be ready in time to combat the current outbreak in West Africa, Dr. Jeffry Turner, president and CEO of Defyrus, says it has the potential to save many lives when the next epidemic strikes. "Unfortunately the outbreaks continue and we're pushing as fast as we can on things." Jaya Rastogi, CMAJ

CMAJ 2014. DOI:10.1503/cmaj.109-4869 